\title{
Low Voltage Imaging and X-Ray Microanalysis in the FE-SEM
}

\author{
Raynald Gauvin
}

Department of Materials Engineering, McGill University, M. H. Wong Bldg, 3610 University Street, Montréal, Québec, Canada, H3A 2B2.

The new generation of Field Emission Scanning Electron Microscope (FE-SEM) can perform high resolution imaging at incident electron beam energy below $1 \mathrm{keV}$. Images with resolution smaller than 2 $\mathrm{nm}$ are now guaranties by various manufacturers. Since imaging below $1 \mathrm{keV}$ allows obtaining surface details of nanomaterials and reduces beam damage for sensitive materials, it is clear that electron microscopy is now entering in a new era. With FE-SEM that can operate in the $50 \mathrm{eV}$ to $30 \mathrm{keV}$ mode with many images modes like conventional bulk secondary electron (SE) or backscattered electron (BSE) imaging or new scanning transmission electron microscopy (STEM) imaging of transparent materials in bright field or dark field mode, the versatility of these microscopes is obvious. Also, if we keep in mind that we can also perform quantitative x-ray microanalysis with start of the art SDD EDS detectors and crystallographic characterization of materials with EBSD detectors, FE-SEM has a very bright future and its importance in science and technology will growth faster than ever because we have now microscopes that can deliver enough current with high spatial resolution to fully exploit the advantages of low voltage scanning electron microscopy as predicted by Von Ardenne as far as 1942. This paper will present new results for the characterization of various nanomaterials obtained with the new Hitachi SU - 8000 cold field FE-SEM recently acquired by the research group of Pr. Gauvin. This FE-SEM has 1 SE lower detector, 2 SE upper detectors with various modes of energy filtration, a five quadrant BSE detector, a STEM detector that works in bright field, an electron convertor that allows to use the SE lower detector for dark field STEM imaging, a $80 \mathrm{~mm} 2$ SDD EDs detector (Oxford Instrument) and the EBSD Nordlys II System (Oxford Instrument). The maximum probe current is $40 \mathrm{nA}$ and this will allow to perform quantitative $\mathrm{x}$-ray microanalysis at low voltage. Also, this will allow acquiring EBSD maps at a faster time, eliminating the problems of drift current issues and flashing. Figure [1] shows pictures of an $\mathrm{Al}_{2} \mathrm{O}_{3}$ sphere taken at $700 \mathrm{eV}$ with the upper top SE detector at a magnification of $250,000 \mathrm{X}$ and $2,000,000 \mathrm{X}$. These spheres were deposited on a Si wafer substrate without coating or sputtering. The picture at $250000 \mathrm{X}$ shows clearly the steps between the crystallographic planes without charging. The picture at $2000000 \mathrm{X}$ shows details with a resolution of $1 \mathrm{~nm}$ which could be clustering of $\mathrm{Al}$ and $\mathrm{O}$ atoms on the crystallographic planes. Impressive image details can therefore now be obtained with start of the art FE-SEM at low beam energy. Since the magnification of these FE-SEM can range between $10 \mathrm{X}$ to $2000000 \mathrm{X}$, it is clear that the understanding of the microstructures of real materials and/or devices will be done extensively with this type of microscope with the combination of EDS and EBSD for quantitative characterization. 


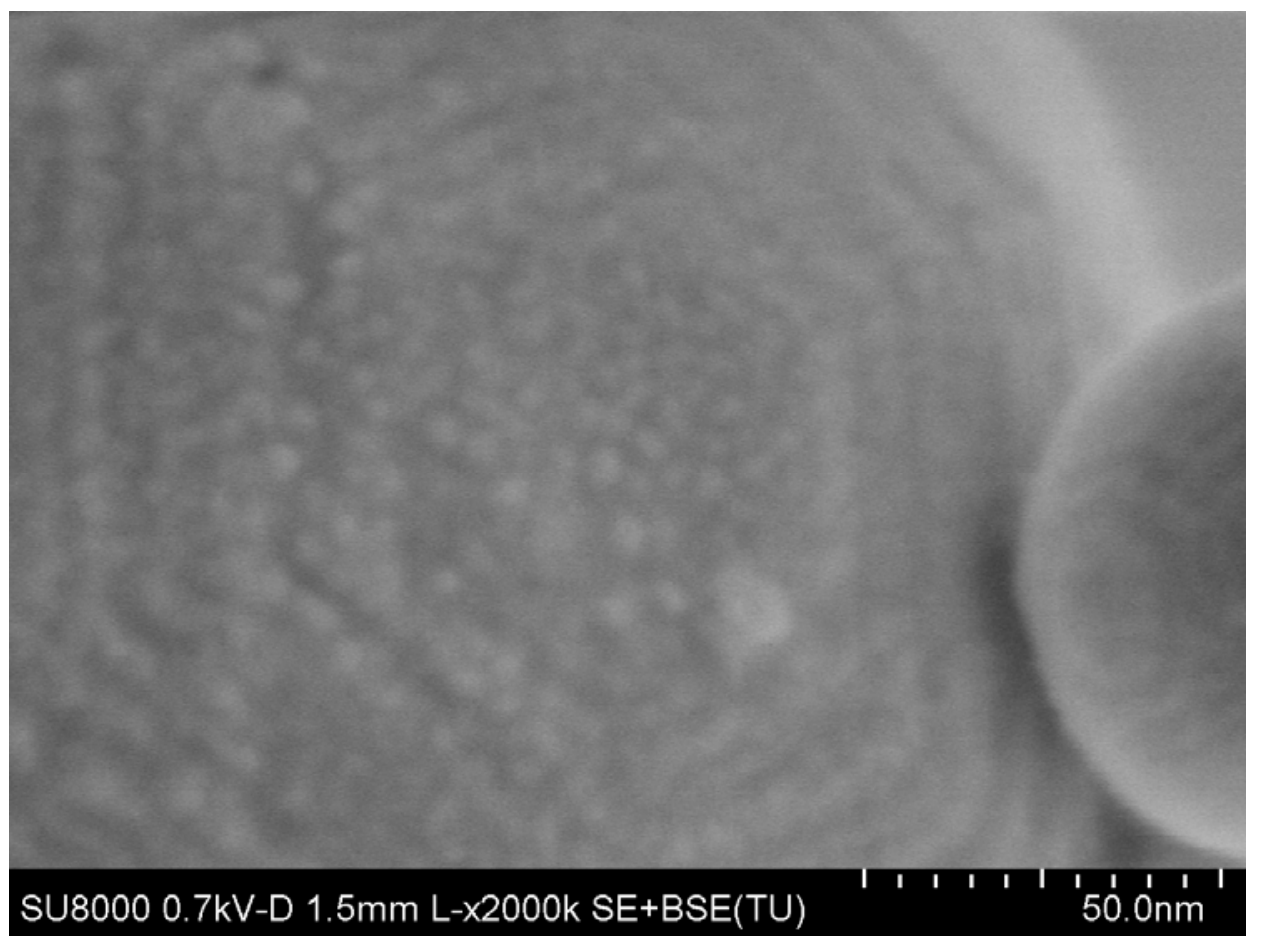

Figure $[1, a] \mathrm{Al}_{2} \mathrm{O}_{3}$ sphere.

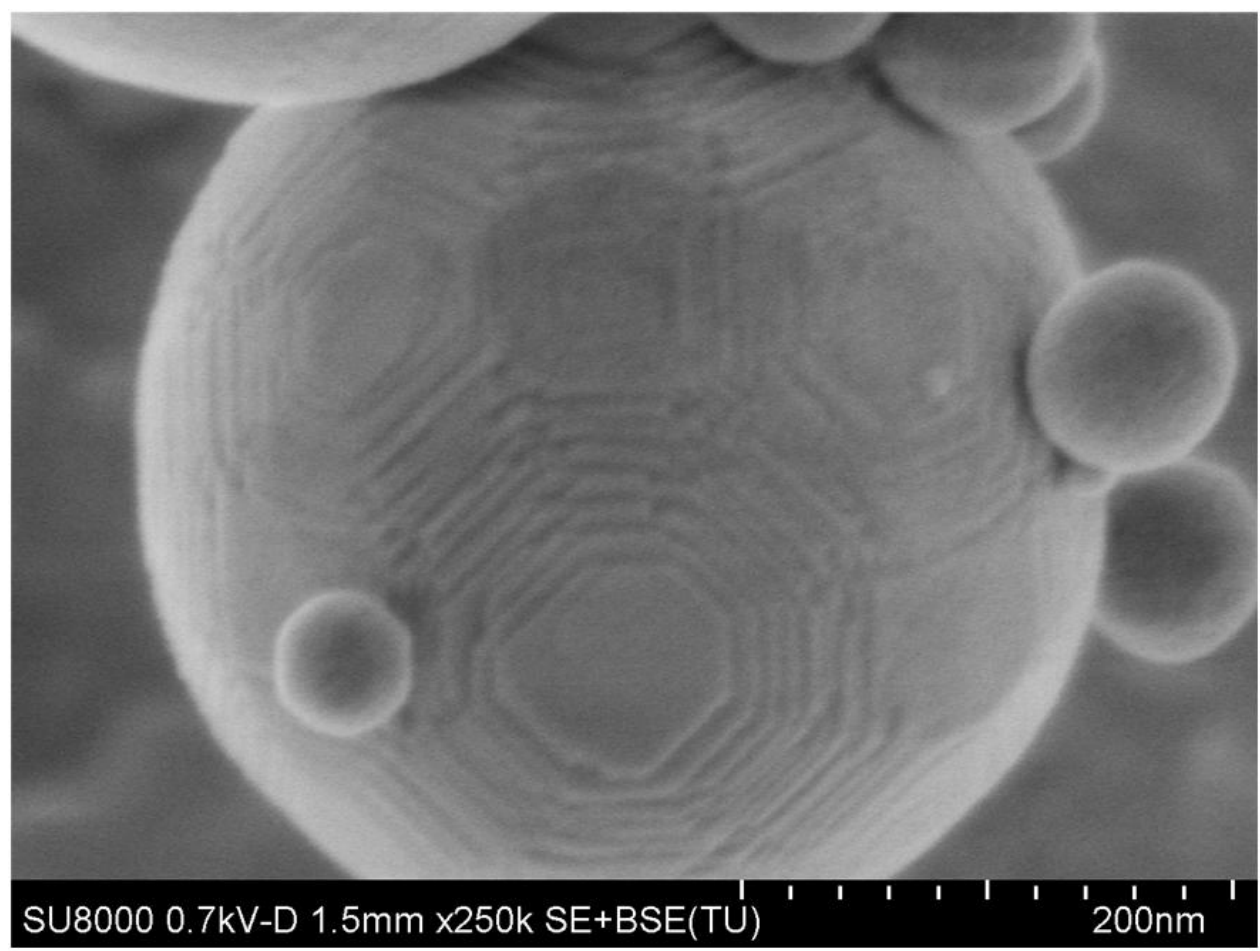

Figure [1, b] Specimen courtesy of Dr. Brendan J. Griffin, University of Western Australia. 